\title{
Imaging findings of uterine adenosarcoma with sarcomatous overgrowth: two case reports, emphasizing restricted diffusion on diffusion weighted imaging
}

Go Nakai ${ }^{1 *}$ (D, Hiroki Matsutani ${ }^{1}$, Takashi Yamada ${ }^{2}$, Masahide Ohmichi ${ }^{3}$, Kazuhiro Yamamoto ${ }^{1}$ and Keigo Osuga ${ }^{1}$

\begin{abstract}
Background: Adenosarcoma is classified as a mixed epithelial and mesenchymal tumor composed of a benign epithelial component and a malignant stromal component. The stromal component in adenosarcoma is usually low grade, and consequently the prognosis is relatively favorable. While, adenosarcoma with sarcomatous overgrowth (SO) is defined as an adenosarcoma in which the sarcomatous component constitutes more than $25 \%$ of the tumor. The stromal component is also high-grade sarcoma showing greater nuclear pleomorphism and mitotic activity, thus, it is associated with worse prognosis. MRI findings of adenosarcoma without SO have been described in previous literatures but the imaging findings in adenosarcoma with SO may be poorly defined. Therefore we present two cases of uterine adenosarcoma with SO.
\end{abstract}

Case presentation: Patient 1 was a 76-year-old woman referred to our hospital with complaint of abdominal distension and postmenopausal bleeding. Patient 2 was a 57-year-old woman with complaint of lower abdominal pain and abnormal uterine bleeding. On magnetic resonance imaging (MRI), T2 weighted imaging showed a large, heterogeneous high-intensity mass with hyperintense tiny cysts that expanded the uterine cavity and extended into the cervical canal for both patients. On diffusion-weighted imaging (DWI), both masses appeared as high signal intensity. Patient 2 also had a right ovarian adult granulosa cell tumor that may have contributed to development of the adenosarcoma. Patient 1 recurred with peritoneal sarcomatosis 6 months after surgery and died of the disease. Patient 2 also recurred with a left upper lung metastasis 3 months after surgery.

Conclusions: DWI may depict pathological changes produced by SO of adenosarcoma as high signal intensity, even though SO does not seem to change MRI findings of adenosarcoma on other sequences. Therefore, DWI could potentially predict SO in presumptive adenosarcoma on MRI and the patient's prognosis. It is also important for pathologists to know if $\mathrm{SO}$ can arise in adenosarcoma because they need to examine the tumor thoroughly to determine the percentage of $\mathrm{SO}$ component in the tumor volume when $\mathrm{SO}$ is present.

Keywords: Magnetic resonance imaging, Adenosarcoma, Sarcomatous overgrowth, Diffusion weighted imaging, Case report

*Correspondence: go.nakai@ompu.ac.jp

${ }^{1}$ The Department of Diagnostic Radiology, Osaka Medical

and Pharmaceutical University, 2-7 Daigaku-machi, Takatsuki City, Osaka

569-8686, Japan

Full list of author information is available at the end of the article

\section{Background}

Adenosarcoma is classified as a mixed epithelial and mesenchymal tumor composed of a benign epithelial component and a malignant stromal component. The stromal 
component in adenosarcoma is usually low grade, and consequently the prognosis is relatively favorable compared with that of other gynecological sarcomas [1]. However, sarcomatous overgrowth (SO), in which the sarcomatous component showing greater nuclear pleomorphism and mitotic activity constitutes more than $25 \%$ of the tumor [2], often results in myometrial invasion and vascular invasion, leading to a worse prognosis [3]. Although MRI findings of adenosarcoma without SO have been described in the literature $[4,5]$, the imaging findings in adenosarcoma with SO may be poorly defined. [6].

\section{Case presentation Case 1}

A 76-year-old woman (para [P] 1) referred to our hospital with a chief complaint of abdominal distension and postmenopausal bleeding for 4 months consulted a gynecologist and was found to have a bulky uterus with a 132-mm subendometrial mass on vaginal ultrasonography. Adenosarcoma was suspected on the basis of findings from biopsy by hysteroscopy. She had no significant past medical history. Serum cancer antigen (CA) 125 and CA 19-9 were $283 \mathrm{U} / \mathrm{ml}$ (normal range $0-35 \mathrm{U} / \mathrm{ml}$ ) and $11.5 \mathrm{U} / \mathrm{ml}$ (normal range $0-35 \mathrm{U} / \mathrm{ml}$ ) respectively.

Magnetic resonance imaging (MRI) was performed at 1.5 Tesra for further examination. Sagittal T2-weighted imaging (WI) (repetition time [TR]/echo time [TE], $4650 / 120 \mathrm{~ms}$ ) showed a large, heterogeneous high-intensity mass, approximately $241 \times 114 \times 88 \mathrm{~mm}^{3}$ in size, without myometrial invasion expanding the uterine cavity and extending into the cervical canal (Fig. 1a). The enlarged uterine size was $250 \times 126 \times 99 \mathrm{~mm}^{3}$. The mass showed low intensity with areas of slightly high signal intensity on fat-suppressed T1WI (TR/TE, 600/10 ms) (Fig. 1b). The mass showed inhomogeneous contrast enhancement with cystic changes of variable sizes and necrotic foci, high intensity on diffusion-weighted imaging (DWI) $\left(b=1000 \mathrm{~s} / \mathrm{mm}^{2}\right.$, TR/TE, 7000/100 ms) (Fig. 1c) and low intensity $\left(1.26 \times 10^{-3} \mathrm{~mm}^{2} / \mathrm{s}\right)$ on apparent diffusion coefficient (ADC) maps. Uterine carcinosarcoma or adenosarcoma was suspected as the preoperative diagnosis.

Total abdominal hysterectomy and bilateral adnexectomy with pelvic lymphadenectomy were subsequently performed.

Benign glandular epithelial components surrounded by atypical stromal cells with a high mitotic rate $(20 / 10$ high power field $[\mathrm{HPF}]$ ) were identified on pathological examination. High-intensity areas observed on T1WI corresponded to hemorrhage, and SO was present. The tumor was diagnosed as adenosarcoma with $\mathrm{SO}$ without myometrial invasion (T1aNOM0).
A follow-up CT scan performed 6 months after surgery revealed peritoneal sarcomatosis. Although her gynecologist recommended adjuvant chemotherapy, she did not wish to receive the treatment and died of the disease 1.5 months after recurrence.

\section{Case 2}

A 57-year-old woman (P 3) with complaint of low abdominal pain and abnormal uterine bleeding for 3 months consulted a gynecologist. Menstrual cycle length ranged from 30 to 40 days. She had no significant past medical history. Endometrial biopsy indicated atypical endometrial hyperplasia. Vaginal ultrasonography showed an enlarged uterus measuring $150 \mathrm{~mm}$ accompanied by a 73-mm tumor in the anterior wall, and both ovaries were not visualized. CA 125 and CA 19-9 were $40.3 \mathrm{U} / \mathrm{ml}$ (normal range $0-35 \mathrm{U} / \mathrm{ml}$ ) and $1.1 \mathrm{U} / \mathrm{ml}$ (normal range $0-35 \mathrm{U} / \mathrm{ml}$ ), respectively. Serum estradiol (E2) was within the normal limit $(130.0 \mathrm{pg} / \mathrm{mL})$.

MRI showed a intrauterine mass, approximately $96 \times 74 \times 57 \mathrm{~mm}^{3}$ in size, protruding into the cervical canal with clearly defined inhomogeneous high intensity on T2WI (TR/TE, 6130/100 ms) (Fig. 2a) and low intensity with a slight high signal intensity area on fatsuppressed T1WI (TR/TE, 575/13 ms). The enlarged uterine size was $129 \times 82 \times 76 \mathrm{~mm}^{3}$.The mass showed inhomogeneous contrast enhancement with cystic changes of variable sizes and necrotic foci (Fig. 2b), high intensity on DWI $\left(b=1000 \mathrm{~s} / \mathrm{mm}^{2}\right.$, TR/TE, 4317/70 ms) (Fig. 2c) and low intensity $\left(0.88 \times 10^{-3} \mathrm{~mm}^{2} / \mathrm{s}\right)$ on ADC maps (Fig. 2d). A $22-\mathrm{mm}$ solid nodule accompanied by a tiny cyst was detected in the right ovary. T2WI showed homogenous iso signal intensity with the uterine tumor on T2WI (Fig. 2a) and low intensity on T1WI. The nodule had homogeneous contrast enhancement with a tiny cystic change (Fig. 2b), high intensity on DWI (Fig. 2c), and low intensity $\left(0.66 \times 10^{-3} \mathrm{~mm}^{2} / \mathrm{s}\right)$ on ADC maps (Fig. 2d).

Whole-body 18F-fluorodeoxyglucose (FDG) positron emission tomography-computed tomography (PET-CT) showed increased FDG uptake (maximum standardized uptake value: 8.2 ) in the uterine tumor and no abnormal FDG uptake in any other organs, including the right ovarian nodule (Fig. 2e). Uterine carcinosarcoma or adenosarcoma metastasizing to the right ovary was suspected as the preoperative diagnosis.

The patient underwent abdominal modified radical hysterectomy with bilateral adnexectomy, pelvic and para-aortic lymphadenectomy, and omentectomy.

Benign glandular epithelial components surrounded by atypical stromal cells with a high mitotic rate (15/10 HPF) with minimal myometrial invasion and $\mathrm{SO}$ were present in the uterine tumor on pathological examination, and 


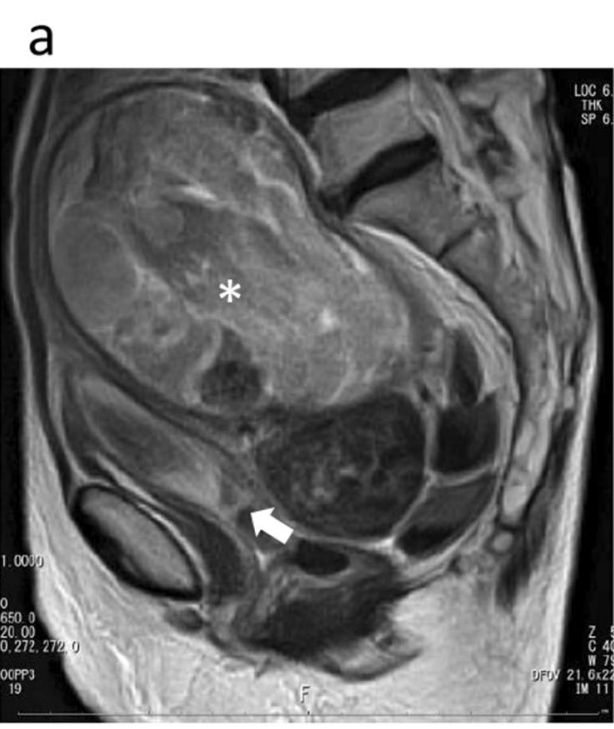

\section{b}

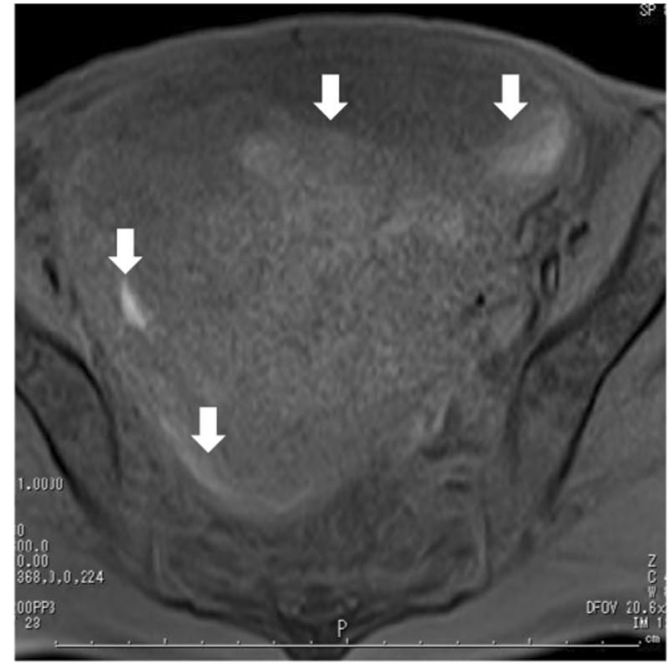

C

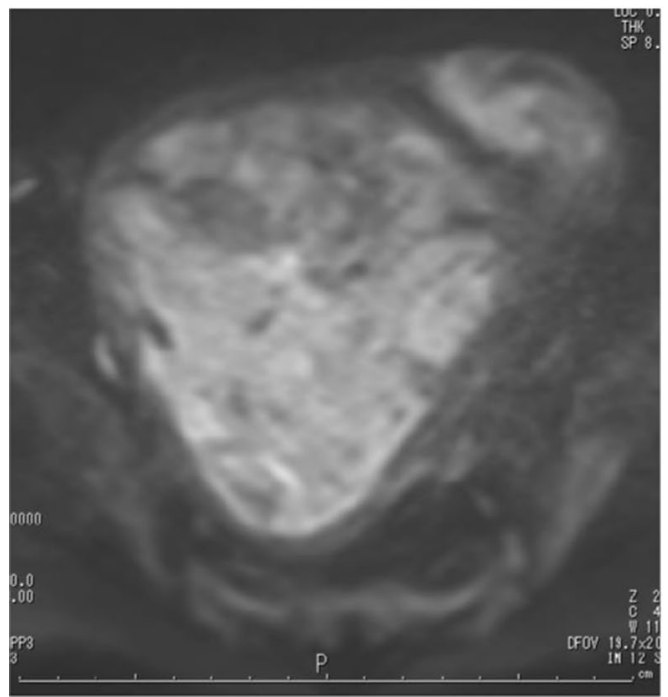

d

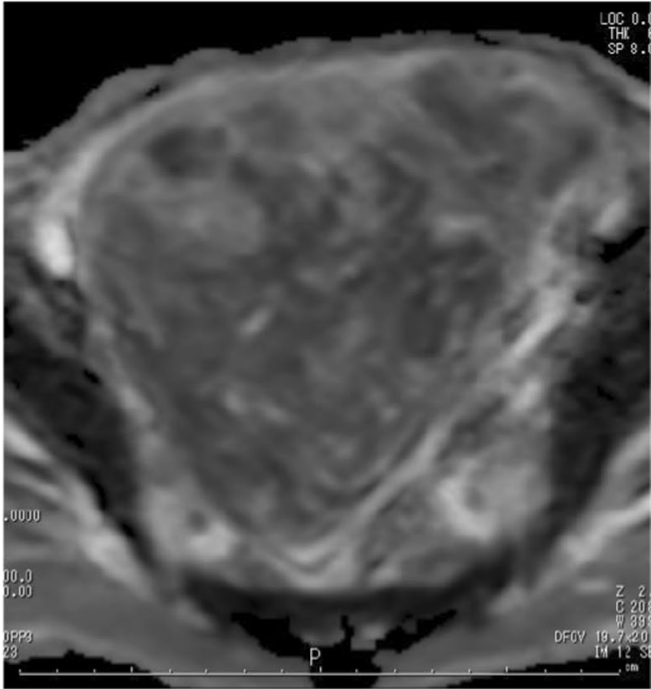

Fig. 1 (a) Sagittal T2-weighted imaging (repetition time [TR]/echo time [TE], 4650/120 ms) showed a large, heterogeneous high-intensity mass without myometrial invasion expanding the uterine cavity $\left(^{*}\right)$ and extending into the cervical canal (arrow). (b) The mass showed low signal intensity with areas of slightly high signal intensity (arrows) on fat-suppressed T1-weighted imaging (TR/TE, 600/10 ms). (c) The mass showed high intensity on diffusion-weighted imaging ( $b=1000 \mathrm{~s} / \mathrm{mm}^{2}$, TR/TE, $\left.7000 / 100 \mathrm{~ms}\right)$ and low intensity $\left(1.26 \times 10^{-3} \mathrm{~mm}^{2} / \mathrm{s}\right)$ on apparent diffusion coefficient maps (d)

(See figure on next page.)

Fig. 2 (a) Axial T2 weighted imaging (TR/TE, 6130/100 ms) showed an intrauterine mass (arrow) with clearly defined inhomogeneous high intensity. (b) The mass (arrow) showed inhomogeneous contrast enhancement with cystic changes of variable sizes on post-contrast fat-suppressed T1 weighted imaging (TR/TE, 575/13 ms), high signal intensity on diffusion-weighted imaging (DWI) (b=1000 s/mm², TR/TE, 4317/70 ms) (c) and low signal intensity $\left(0.88 \times 10^{-3} \mathrm{~mm}^{2} / \mathrm{s}\right)$ on apparent diffusion coefficient (ADC) maps (d). A 22-mm solid nodule accompanied by a tiny cyst was detected in the right ovary (a-d arrowhead). The nodule showed homogenous iso signal intensity with the uterine tumor on T2WI (a). It showed homogeneous contrast enhancement with a tiny cystic change (b), marked high signal intensity on DWI (c) and low signal intensity $\left(0.66 \times 10^{-3} \mathrm{~mm}^{2} / \mathrm{s}\right)$ on ADC maps (d). Whole-body $18 \mathrm{~F}$-FDG positron emission tomography-computed tomography (PET-CT) showed increased FDG uptake (maximum standardized uptake value: 8.2) in the uterine tumor (arrow) and no abnormal FDG uptake in any other organs, including the right ovarian nodule (e arrowhead) 


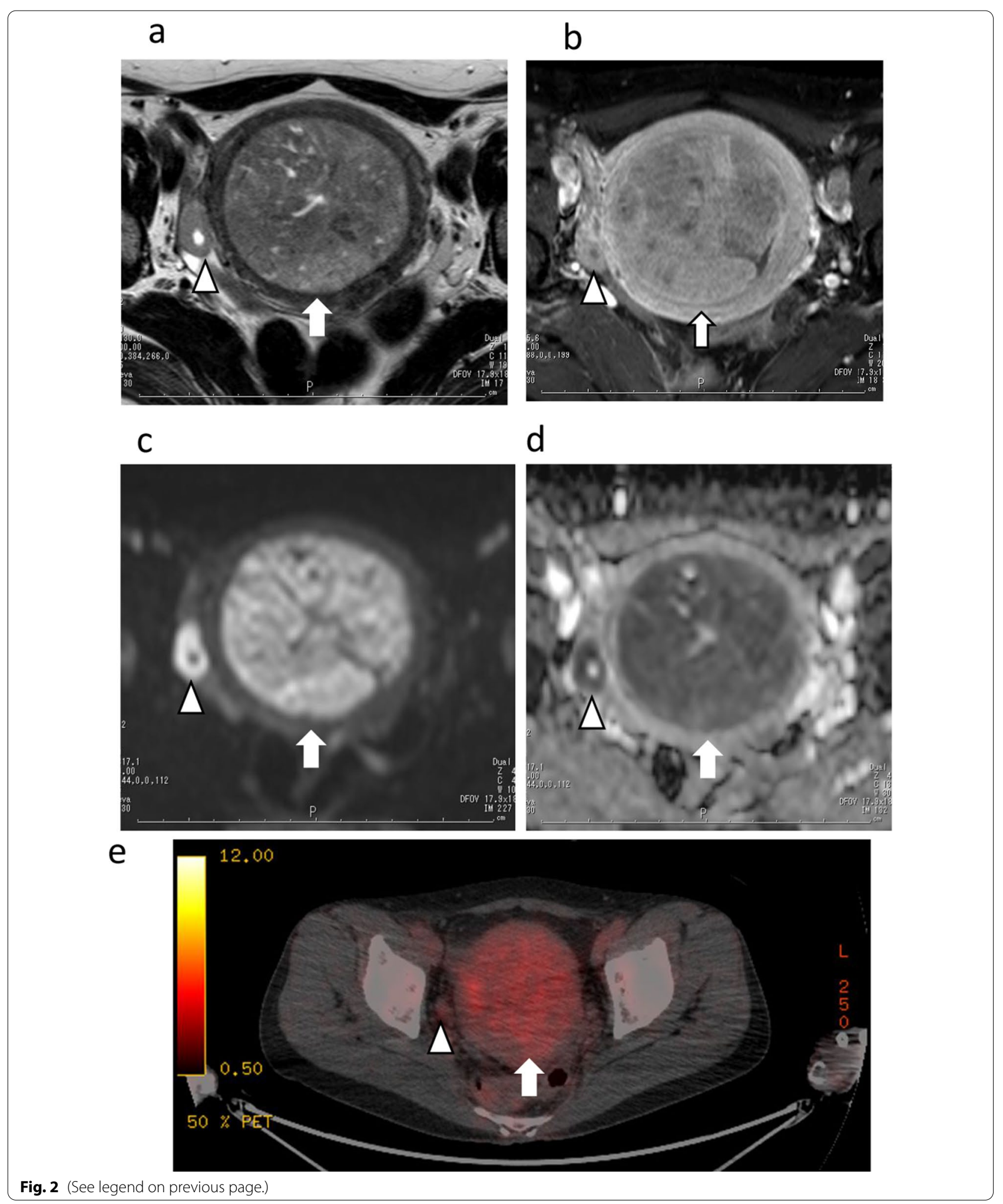


thus the tumor was diagnosed as adenosarcoma with $\mathrm{SO}$ (T1bN0M0). The right ovarian nodule was diagnosed as an adult granulosa cell tumor (GCT).

A follow-up CT scan revealed a left upper lung metastasis 3 months after surgery. Accordingly, the patient underwent thoracoscopic segmentectomy of the left upper lobe. As of the writing of this report, at 22 months after the surgery, she has showed no signs of recurrence.

\section{Discussion and conclusions}

Adenosarcoma with $\mathrm{SO}$ is defined as an adenosarcoma in which the sarcomatous component constitutes more than $25 \%$ of the tumor [2]. The stromal component is also high-grade sarcoma showing greater nuclear pleomorphism and mitotic activity, leading to a worse prognosis. Recurrence was observed within 6 months after surgery in both of our patients and one died of the disease, probably due to its aggressive clinical behavior and disease progression.

MRI findings of adenosarcoma have been previously described in the literature $[4,5,7]$. It typically presents as a solitary, exophytic, polypoid mass in the endometrial cavity that protrudes through the cervical os. The mass consists of heterogeneous solid components with tiny cysts corresponding to glandular cavities that show high signal intensity on T2WI. The solid component shows heterogenous high signal intensity compared to the myometrium on T2WI and low- or iso-intensity compared to the myometrium on gadolinium-enhanced T1WI. Hemorrhagic necrosis showing high signal intensity on T1WI is common, particularly when SO is present. These MRI findings are common irrespective of the presence of SO, and are consistent with MRI findings in our patients. However, only two previous reports have described DWI findings of adenosarcoma. Fujii et al. reported a case of adenosarcoma without $\mathrm{SO}$ arising from adenomyosis showing slightly high signal intensity on DWI and high intensity $\left(1.71 \times 10^{-3} \mathrm{~mm}^{2} / \mathrm{s}\right)$ on ADC maps. Benign glandular epithelial components surrounded by atypical stromal cells with mitosis $(2-3 / 10 \mathrm{HPF})$ were identified on pathological examination [7]. Takeuchi et al. reported uterine low-grade adenosarcoma without a marked signal increase throughout the tumor on DWI. As this contrasts with the marked increase in signal on DWI that we observed in our patients who had adenosarcoma with $\mathrm{SO}$, we assume the presence of SO is associated with increasing signal intensity on DWI.

The diagnosis of adenofibroma was removed from the WHO Classification of Tumours, 5th Edition, Volume 4 [8], and it is believed that the majority of tumors previously diagnosed as adenofibroma were simply low-grade adenosarcomas or endometrial or cervical polyps with unusual morphology. Endometrial polyps usually show relatively lower signal intensity than malignant endometrial tumors on DWI [9] because they are composed of irregularly shaped glands with fibrous stroma, resulting in lower cellularity than highly cellular malignant tumors. The stromal component in adenosarcoma is usually low grade, and thus uterine cervical adenosarcoma initially diagnosed as a cervical polyp pathologically can present as a recurrent cervical polyp [10]. Consequently, it may occasionally be challenging to differentiate adenosarcoma from polyps even pathologically, as polyps may have cellular stroma and architectural features that overlap to some degree with those of adenosarcoma [11]. Given these similarities in pathologic features between adenosarcomas and polyps, the reported signal intensity of adenosarcoma without SO on DWI is consistent with its pathological features, whereas $\mathrm{SO}$ showing greater nuclear pleomorphism and mitotic activity might result in markedly high signal intensity on DWI as seen in our patients. Therefore, DWI could potentially be used to predict SO in presumptive adenosarcoma on MRI and the patient's prognosis. It is also important for pathologists to know if SO can arise in adenosarcoma because they need to examine the tumor thoroughly to determine the percentage of $\mathrm{SO}$ component in the tumor volume when $\mathrm{SO}$ is present.

Although tumor size is not regarded as important in the diagnosis of adenosarcoma, it is important to note that mean size of polyps $(1.4-2.5 \mathrm{~cm})$ is much smaller than that of adenosarcoma $(6.5 \mathrm{~cm})[9,11]$. Large tumor size (major axis $>10 \mathrm{~cm}$ ) is not a good indicator of $\mathrm{SO}$ either because many tumors without $\mathrm{SO}$ are also large $[4$, $5,12]$, although high mitotic rate seems to be associated with large tumor size.

FDG PET-CT findings in uterine adenosarcoma have never been reported. However, there has been one report of FDG PET-CT findings of recurrent tumors in a patient with uterine cervical adenosarcoma with SO [13]. According to that report, recurrent tumors in the vaginal vault, right pelvic lymph nodes and pubic ramus showed intense FDG uptake, although preoperative PET-CT was not performed. Given the intense FDG uptake in the primary lesion in Patient 2 and the metastatic lesions in the previous report, the right ovarian nodule in Patient 2 which showed high signal intensity on DWI without any FDG uptake on PET-CT should not have been regarded as a metastasis. According to previous reports describing imaging findings of GCTs, they showed mild FDG uptake on PET-CT and restricted diffusion (high signal intensity) on DWI $[14,15]$, although FDG uptake of GCT may vary widely due to its variety of clinical features and uncertain potential of malignancy [16, 17]. In Patient 2, E2 continuously produced by the right ovarian GCT might be one of the causes of the uterine adenosarcoma with SO because 
studies have shown an association with long-term unopposed oestrogen therapy such as tamoxifen therapy [12, 18 .

In conclusion, DWI may depict pathological changes produced by $\mathrm{SO}$ of adenosarcoma as high signal intensity, even though SO does not seem to change MRI findings of adenosarcoma on other sequences. Therefore, DWI could potentially predict $\mathrm{SO}$ in presumptive adenosarcoma on MRI and the patient's prognosis. It is also important for pathologists to know if $\mathrm{SO}$ can arise in adenosarcoma because they need to examine the tumor thoroughly to determine the percentage of $\mathrm{SO}$ component in the tumor volume when $\mathrm{SO}$ is present.

\section{Abbreviations}

SO: Sarcomatous overgrowth; G: Gravida; P: Para; CA: Cancer antigen; MRI: Magnetic resonance imaging; WI: Weighted imaging; TR: Repetition time; TE: Echo time; DWl: Diffusion-weighted imaging; ADC: Apparent diffusion coefficient; HPF: High power field; E2: Estradiol; FDG: Fluorodeoxyglucose; PET-CT: Positron emission tomography-computed tomography; GCT: Adult granulosa cell tumor.

\section{Acknowledgements}

Not applicable.

\section{Authors' contributions}

GN contributed to writing the manuscript. HM contributed to corrected patients' data for the work. TY performed postoperative histological examination. MO contributed to the patients' operation. KY and KO contributed to revising the manuscript. All authors read and approved the final manuscript.

\section{Funding}

No funding.

\section{Availability of data and materials}

The datasets used and/or analyzed during the current study are available from the corresponding author on reasonable request.

\section{Declarations}

Ethics approval and consent to participate

Not applicable.

\section{Consent for publication}

Written informed consent was obtained from the patient for publication of this case report, any associated images and clinical details.

\section{Competing interests}

The authors declare that they have no competing interests.

\section{Author details}

${ }^{1}$ The Department of Diagnostic Radiology, Osaka Medical and Pharmaceutical University, 2-7 Daigaku-machi, Takatsuki City, Osaka 569-8686, Japan. ${ }^{2}$ The Department of Pathology, Osaka Medical and Pharmaceutical University, 2-7 Daigaku-machi, Takatsuki City, Osaka 569-8686, Japan. ${ }^{3}$ The Department of Obstetrics and Gynecology, Osaka Medical and Pharmaceutical University, 2-7 Daigaku-machi, Takatsuki City, Osaka 569-8686, Japan.

Received: 21 August 2021 Accepted: 10 December 2021 Published online: 16 December 2021
References

1. Arend R, Bagaria M, Lewin SN, Sun X, Deutsch I, Burke WM, Herzog TJ, Wright JD. Long-term outcome and natural history of uterine adenosarcomas. Gynecol Oncol. 2010;119(2):305-8.

2. Clement PB, Scully RE. Mullerian adenosarcoma of the uterus: a clinicopathologic analysis of 100 cases with a review of the literature. Hum Pathol. 1990;21(4):363-81.

3. Kaku T, Silverberg SG, Major FJ, Miller A, Fetter B, Brady MF. Adenosarcoma of the uterus: a gynecologic oncology group clinicopathologic study of 31 cases. Int J Gynecol Pathol. 1992;11(2):75-88.

4. Yoshizako T, Wada A, Kitagaki H, Ishikawa N, Miyazaki K. MR imaging of uterine adenosarcoma: case report and literature review. Magn Reson Med Sci. 2011;10(4):251-4.

5. Takeuchi M, Matsuzaki K, Yoshida S, Kudo E, Bando Y, Hasebe H, Kamada M, Nishitani H. Adenosarcoma of the uterus: magnetic resonance imaging characteristics. Clin Imaging. 2009;33(3):244-7.

6. Wang B, Yang HD, Shi XH, Li H. Advanced uterine adenosarcoma with sarcomatous overgrowth in a young woman: a case report. Medicine (Baltimore). 2019;98(47):e18119.

7. Fujii S, Nosaka K, Mukuda N, Fukunaga T, Sato S, Ogawa T. MR imaging of an intramural adenosarcoma with pathologic correlation. Magn Reson Med Sci. 2018;17(1):1-2.

8. Board WHOcote, World Health Organization. Female genital tumours, vol. 5. v. 4. World Health Organization, International Agency for Research on Cancer; 2020

9. Hase S, Mitsumori A, Inai R, Takemoto M, Matsubara S, Akamatsu N, Fujisawa M, Joja I, Sato S, Kanazawa S. Endometrial polyps: MR imaging features. Acta Med Okayama. 2012;66(6):475-85.

10. Charfi S, Kallel R, Mnif H, Ellouze S, Dhouib M, Guermazi M, Khabir A, Sellami-Boudawara T. Mullerian adenosaroma of the cervix with sarcomatous overgrowth and heterologous elements presenting as a recurrent cervical polyp. Case Rep Obstet Gynecol. 2012;2012:358302.

11. Howitt BE, Quade BJ, Nucci MR. Uterine polyps with features overlapping with those of Mullerian adenosarcoma: a clinicopathologic analysis of 29 cases emphasizing their likely benign nature. Am J Surg Pathol. 2015;39(1):116-26

12. Soh E, Eleti A, Jimenez-Linan M, Arends MJ, Latimer J, Sala E. Magnetic resonance imaging findings of tamoxifen-associated uterine Mullerian adenosarcoma: a case report. Acta Radiol. 2008;49(7):848-51.

13. Morales FD, Medina RM, Trujillo LM, Beltran MI, Dulcey IC. Mullerian adenosarcoma of the uterine cervix with sarcomatous overgrowth: a case report of aggressive disease in a young patient. Int I Surg Case Rep. 2016;27:155-61.

14. Günyeli I, Bozkurt KK, Yalçın Y, Tatar B, Cerçi SS, Erdemoğlu E. Granulosa cell tumor and concurrent endometrial cancer with (18)F-FDG uptake. Hell J Nucl Med. 2014;17(2):153-5.

15. Zhang H, Zhang H, Gu S, Zhang Y, Liu X, Zhang G. MR findings of primary ovarian granulosa cell tumor with focus on the differentiation with other ovarian sex cord-stromal tumors. J Ovarian Res. 2018;11(1):46.

16. Caoduro C, Ungureanu CM, Singeorzan CM, Angoue O, Blagosklonov O, Boulahdour H. Granulosa cell tumor of the ovary with high FDG uptake. Clin Nucl Med. 2013;38(7):553-6.

17. Tanizaki Y, Kobayashi A, Shiro M, Ota N, Takano R, Mabuchi Y, Yagi S, Minami S, Terada M, Ino K. Diagnostic value of preoperative SUVmax on FDG-PET/CT for the detection of ovarian cancer. Int J Gynecol Cancer. 2014;24(3):454-60.

18. Clement PB, Oliva E, Young RH. Mullerian adenosarcoma of the uterine corpus associated with tamoxifen therapy: a report of six cases and a review of tamoxifen-associated endometrial lesions. Int J Gynecol Pathol. 1996;15(3):222-9.

\section{Publisher's Note}

Springer Nature remains neutral with regard to jurisdictional claims in published maps and institutional affiliations. 